\title{
Eficiencia en el deporte: Entrenadores en la Primera División del fútbol español 2009-2011
}

\author{
JULIO DEL CORRAL \\ Departamento de Análisis Económico y Finanzas, UNIVERSIDAD DE CASTILLA-LA \\ MANCHA, ESPAÑA. E-mail: julio.corral@uclm.es
}

\begin{abstract}
RESUMEN
Para calcular la eficiencia de los entrenadores en una liga deportiva es necesario conocer las medidas del output e inputs. Los puntos obtenidos en la liga suelen utilizarse como output, mientras que como inputs se utilizan variables que aproximen la calidad de los jugadores de la plantilla. Este trabajo utiliza los precios de los jugadores en el juego Comunio como medida de la calidad de estos. Para calcular la eficiencia de los entrenadores de la Primera División del fútbol español en las temporadas 2009/2010 y 2010/2011 se estima una frontera estocástica en donde se obtiene que los entrenadores que han sido jugadores profesionales son menos eficientes que los entrenadores que no fueron futbolistas profesionales.
\end{abstract}

Palabras clave: Eficiencia técnica, entrenadores, frontera estocástica, fútbol.

\section{Efficiency in Sports: Coaches in the Spanish Football First Division 2009-2011}

\begin{abstract}
In order to calculate the managers' efficiency of team sports within a league is necessary to know the output and inputs. The points obtained in the league are usually used as output, while as input are usually used variables that engage the quality of the rooster. In this paper the players' prices from a football fantasy league are used as a measure of the players' quality. A stochastic frontier is estimated to calculate the efficiency of the coaches in the Spanish First Division in the 2009/2010 and 2010/2011 seasons. The results show that the coaches that have been professional players are less efficient that coaches that did not.
\end{abstract}

Keywords: Technical Efficiency, Managers, Stochastic Frontier, Football.

Clasificación JEL: C13, L83

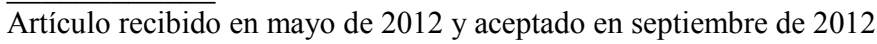

Artículo disponible en versión electrónica en la página www.revista-eea.net, ref. ə-30218 


\section{INTRODUCCIÓN}

Las técnicas frontera son cada vez más habituales en la literatura de la Economía del Deporte. Mientras que las técnicas econométricas clásicas como Mínimos Cuadrados Ordinarios estiman la relación existente entre las variables endógenas y exógenas en la media de los datos las técnicas frontera se caracterizan por estimar la relación que existe entre las variables endógenas y exógenas para las observaciones con mejores resultados. Es decir son funciones que envuelven a los datos bien por la parte superior o inferior de los datos. Esta noción de frontera permite reconciliar el análisis empírico de la producción con la teoría económica, ya que las funciones de producción (i.e., máximo nivel de output que se puede producir dado el nivel de inputs) y coste (i.e., mínimo coste en el que hay que incurrir para producir una determinada cantidad de output dado el precio de los factores productivos) son funciones frontera (Álvarez, 2001, p. 28). Las observaciones que se sitúan en la frontera son llamadas eficientes mientras que las observaciones por debajo (encima en el caso función de costes) se las considera ineficientes. La magnitud de la ineficiencia vendrá dada por la distancia entre el valor observado de la variable dependiente para una observación y el valor de la función frontera para esa observación. Cuanto mayor sea la distancia mayor será la ineficiencia.

El análisis de la eficiencia de los equipos deportivos está teniendo un gran auge. Varios son los aspectos que han sido tratados en esta literatura. Algunos trabajos han intentado evaluar la eficiencia de los equipos usando tanto un análisis primal (González-Gómez y Picazo-Tadeo, 2010, Barros y García-del-Barrio, 2011;) como un análisis dual (Barros y Leach, 2007; Barros, del Corral y García-del-Barrio, 2008). Otros trabajos han evaluado la eficiencia de los equipos en transformar determinadas situaciones del juego en victorias. Carmichael, Thomas y Ward (2001) y Sala et al. (2009) en fútbol, Carmichael y Thomas (1995) en rugby, Zak, Huang y Siegfried (1979) y Hofler y Payne (1997) en baloncesto, Porter y Scully (1982) en béisbol, Hadley et al. (2000) y Collier, Johnson y Ruggiero (2011) en fútbol americano.

Otra rama de esta literatura aborda la eficiencia de los entrenadores. Lee (2006) clasifica esta literatura en función de los modelos econométricos utilizados (i.e., paramétricos y no paramétricos) y de las variables de input utilizadas (i.e., ex ante y ex post). Lee y Berri (2008) y Dawson, Dobson y Gerrard (2000a) sostienen que para calcular la eficiencia de los entrenadores correctamente es necesario utilizar una medida del talento de los jugadores ex ante. Varios trabajos han utilizado una medida del talento de los jugadores ex ante. Por ejemplo, Fizel y D'Itri (1997) utilizaron el índice Hoop Scoop, que es un índice del talento de los jugadores de fútbol americano, para calcular la eficiencia de los entrenadores de las universidades americanas. Este índice se elaboraba cuando un jugador llegaba a la universidad en función de sus estadísticas 
en el instituto y se actualizaba en función del año de universidad en el que se encontrase el jugador o si el jugador mejoraba notablemente en la universidad. Lee y Berri (2008) y Fort, Lee y Berri (2008) calcularon una medida para los jugadores de la NBA por posiciones usando las estadísticas previas de los jugadores para calcular la eficiencia de los entrenadores de la NBA. Gerrard (2001) utilizó el método de los precios hedónicos para obtener un hipotético precio de traspaso. Esta idea fue utilizada en Dawson, Dobson y Gerrard (2000a, 2000b) para analizar la eficiencia de los entrenadores del fútbol inglés. Muchos trabajos (Einolf, 2004; Kahane, 2005; de Dios Tena y Forrest, 2007; Frick y Simmons, 2008; Kern y Süssmuth, 2008; Volz, 2009) han usado los salarios pagados a los deportistas como medida del talento deportivo para calcular índices de eficiencia de los entrenadores. Otros trabajos (González-Gómez, Picazo-Tadeo y García-Rubio, 2011) han usado el presupuesto del equipo como medida de la calidad de la plantilla dado que el porcentaje que supone los salarios respecto a otros gastos es muy alto (en torno al 70\%).

El uso de los salarios como medida de talento deportivo presenta varios problemas: 1. Los salarios de los jugadores profesionales tiene dos componentes, uno deportivo y otro extradeportivo. Éste último reside en la capacidad de los jugadores de generar ingresos para el club a través de su imagen. Cuánto mayor sea el segundo componente el uso del salario como medida de la calidad deportiva será menos apropiado. 2. El salario como cualquier precio de un bien exclusivo depende de la capacidad negociadora de las partes. Es bien conocido que la capacidad negociadora de los futbolistas en España aumenta en relación inversa con el tiempo que le resta de contrato. La idea es bien sencilla, en el caso extremo de cuando un jugador no tiene contrato cualquier equipo puede contratarlo sin tener que pagar traspaso. Por tanto el club que quiere adquirir sus servicios estará dispuesto a pagar un mayor salario a ese jugador dado que no tiene que pagar traspaso. 3. El salario puede ser endógeno, dado que en general los salarios que pagan los clubes dependen en cierta medida de lograr los objetivos deportivos del club. 4. Muy relacionado con el punto anterior los clubes pueden tener estructura salarial diferente en el sentido que algunos clubes preferirán tener un esquema muy alto de salario fijo y bajos salarios variables en función del rendimiento deportivo mientras que otros clubes preferirán lo contrario. 5. Por último, los contratos de los futbolistas suelen ser de larga duración (4 o 5 años). En general el salario es mucho más flexible al alza que a la baja, que no lo es. En general si un jugador está teniendo un rendimiento superior del que se le supone por su salario el club suele elevar el salario a cambio de más años de contrato pero cuando un jugador está teniendo un rendimiento muy inferior al esperado seguirá cobrando el mismo salario.

Una alternativa a las medidas utilizadas en la literatura es emplear el valor de los jugadores en una liga fantástica. Existen básicamente dos tipos de ligas fantásticas, en una el valor de los jugadores es fijado al comienzo del juego por un 
panel de expertos y permanece fijo a lo largo de toda la temporada (e.g., La Liga Fantástica de Marca). Mientras que en otras ligas fantásticas el precio de los jugadores es fijado al comienzo pero se permite comprar y vender jugadores entre los participantes al precio fijado entre ellos. De esta forma se establece un mercado de jugadores, en donde el precio de los jugadores lo establece la ley de oferta y demanda. Este es el caso del Comunio. En este trabajo se utilizan los precios de los jugadores en el Comunio al inicio de la liga como input de los entrenadores. ${ }^{1}$ De esta forma es posible analizar la eficiencia de los entrenadores del fútbol español en las temporadas 2009-2010 y 2010-2011. Para analizar los determinantes de la eficiencia de los entrenadores se estima un modelo de frontera estocástica que permite incorporar determinantes de la eficiencia. En concreto se analizan dos: si el equipo tiene un filial en al menos la tercera división del fútbol español $\left(2^{\mathrm{a}} \mathrm{B}\right)$ y si el entrenador ha sido futbolista profesional.

\section{REVISIÓN DE LOS ANÁLISIS DE EFICIENCIA EN EL FÚTBOL ESPAÑOL}

Varios son los trabajos que han utilizado técnicas frontera con datos del fútbol español. La Tabla 1 muestra una lista con algunas características de los trabajos que han utilizado el enfoque primal. Todos estos trabajos tienen en común que han utilizado el Análisis Envolvente de Datos, que es una técnica no paramétrica.

Tabla 1

Artículos que han estimado funciones de producción con datos del fútbol español

\begin{tabular}{|c|c|c|}
\hline Artículo & Inputs & Outputs \\
\hline $\begin{array}{l}\text { Espitia-Escuer y García } \\
\text { Cebrián (2004) }\end{array}$ & Movimientos atacantes y defensivos & Número de puntos \\
\hline García-Sánchez (2007) & Habilidad defensiva y ofensiva & Número de puntos \\
\hline Giner y Muñoz (2008) & $\begin{array}{l}\text { Consumos de explotación, gastos de personal, } \\
\text { amortización inmovilizado, otros gastos de } \\
\text { explotación, gastos extraordinarios }\end{array}$ & $\begin{array}{l}\text { Ingresos totales, inverso de } \\
\text { la clasificación final }\end{array}$ \\
\hline Boscá et al. (2009) & Disparos y movimientos atacantes y defensivos & Goles marcados y encajados \\
\hline Sala et al. (2009) & Disparos y movimientos atacantes y defensivos & Goles marcados y encajados \\
\hline $\begin{array}{l}\text { Picazo-Tadeo y González- } \\
\text { Gómez (2010) }\end{array}$ & $\begin{array}{l}\mathrm{N}^{\circ} \text { de jugadores, espectadores, temporadas en } \\
\text { Primera }\end{array}$ & Puntos liga \\
\hline $\begin{array}{l}\text { González-Gómez y Picazo- } \\
\text { Tadeo (2010) }\end{array}$ & $\begin{array}{l}\mathrm{N}^{0} \text { de jugadores, espectadores, temporadas en } \\
\text { Primera, trofeos internacionales }\end{array}$ & $\begin{array}{l}\text { Puntos liga, rondas Copa del } \\
\text { Rey, partidos en Europa }\end{array}$ \\
\hline $\begin{array}{l}\text { Barros y García-del Barrio } \\
\text { (2011) }\end{array}$ & $\begin{array}{l}\text { Costes de explotación, activos, gastos en } \\
\text { plantilla }\end{array}$ & $\begin{array}{l}\text { Ingresos por taquilla, otros } \\
\text { ingresos }\end{array}$ \\
\hline $\begin{array}{l}\text { González-Gómez, Picazo- } \\
\text { Tadeo y García-Rubio (2011) }\end{array}$ & Presupuesto, espectadores & $\begin{array}{l}\text { Número de puntos, partidos } \\
\text { extra }\end{array}$ \\
\hline
\end{tabular}

Nota: Todos estos artículos han utilizado metodología DEA.

Fuente: Elaboración propia.

\footnotetext{
${ }^{1}$ Aunque usar el valor del Comunio para valorar la calidad de la plantilla puede ser una buena aproximación a ésta no está exenta de problemas dado el componente subjetivo de ellas.
} 
Giner y Muñoz (2008) y Barros y García-del-Barrio (2011) analizan la eficiencia de los clubes desde un punto de vista financiero. Para ello utilizan variables financieras tanto desde un punto de vista de los inputs como de los outputs. Los trabajos Espitia-Escuer y García-Cebrián (2004), García-Sánchez (2007), Boscá et al. (2009), Sala et al. (2009) analizan la eficiencia de los equipos en transformar determinadas situaciones del juego en victorias. Para ellos utilizan como inputs habilidades medidas ex post. Por tanto, como argumenta Lee (2006) no se está evaluando la eficiencia de los entrenadores al no usar indicadores de la habilidad ex ante. Por último los trabajos de Andrés Picazo y coautores intentan responder a tres preguntas interesantes. En Picazo-Tadeo y González-Gómez (2010) se preguntan si jugar partidos en otras competiciones afecta al resultado en la liga. Sus resultados sugieren que si. En González-Gómez y Picazo-Tadeo proponen utilizar un índice de eficiencia técnica como medida de felicidad con un equipo. Por último en González-Gómez, PicazoTadeo y García-Rubio (2011) obtienen que los entrenadores más eficientes son aquellos que están en el cargo durante toda la temporada mientras que los más ineficientes son los entrenadores despedidos. La diferencia entre la eficiencia de los entrenadores despedidos y la eficiencia de los entrenadores contratados a mitad de temporada resulta negativa y significativa.

Barros, del Corral y García-del-Barrio (2008) estimaron una función de costes utilizando un modelo de frontera estocástica. De forma poco sorprendente encontraron que los equipos más ineficientes en el período analizado fueron el Atlético de Madrid y el Real Betis. De esta revisión de la literatura se desprende que no existe ningún artículo del fútbol español donde se analice la eficiencia de los entrenadores de fútbol. Este artículo pretende rellenar esta ausencia en la literatura.

\section{METODOLOGÍA}

En este trabajo se va a estimar una frontera de producción estocástica. Los modelos de frontera estocástica se empezaron a desarrollar a finales de la década de los setenta (Aigner, Lovell y Schmidt, 1977; Meeusen y van den Broeck, 1977) ${ }^{2}$. Una función de producción frontera estocástica puede escribirse como:

$$
\ln y=f(x)+\varepsilon ; \quad \varepsilon=v-u
$$

donde $y$ representa el output de cada entrenador, $x$ es un vector de inputs, $f(x)$ es la representación de la tecnología, y $\varepsilon$ es una perturbación aleatoria compuesta por dos términos de error: uno simétrico, $v$, que refleja la aleatoriedad del

\footnotetext{
${ }^{2}$ Una revisión de esta literatura puede verse en Álvarez (2001), mientras que una aplicación interesante es Dios Palomares et al. (2003).
} 
proceso productivo como pueden ser la influencia de los arbitrajes, lesiones o sanciones y que se supone que se distribuye como una normal con esperanza cero y desviación típica $\sigma_{v}$, y otro asimétrico, $u$, que recoge la ineficiencia de los entrenadores. El término de ineficiencia es no-negativo, por lo que $u \geq 0$ y se asume que se distribuye como una distribución seminormal ${ }^{3}$. Además, se supone que los componentes $u$ y $v$ son independientes. Con estos supuestos se puede estimar la tecnología mediante la técnica de máxima verosimilitud (Kumbhakar y Lovell, 2000).

Un índice de eficiencia técnica (ET) puede calcularse como la ratio entre el output observado $(y)$ y el máximo output posible $\left(y^{*}\right)$ :

$$
\mathrm{TE}_{\mathrm{i}}=\frac{y_{i}}{y_{i} *}=\frac{f\left(x_{i} ; \beta\right) \cdot \exp \left(v_{i}-u_{i}\right)}{f\left(x_{i} ; \beta\right) \cdot \exp \left(v_{i}\right)}=\exp \left(-u_{i}\right)
$$

Dado que $y$ es siempre menor o igual que $y^{*}$, el índice de eficiencia técnica está comprendido entre 0 y 1 . El índice de eficiencia técnica será de 1 para un entrenador que consiga el mayor número de puntos posible (i.e., $y=y^{*}$ ) dada la calidad de la plantilla de la que dispone. De la estimación del modelo 1, se obtiene directamente el error compuesto $(\varepsilon)$ pero no el valor de la ineficiencia $(u)$. Para obtener el valor de la ineficiencia dado el error compuesto hay que utilizar la fórmula desarrollada por Jondrow et al. (1982):

$$
\mathrm{E}\left[u_{i} \mid \varepsilon_{i}\right]=\frac{\sigma_{u} \sigma_{v}}{\sigma}\left[\frac{g\left(\varepsilon_{i} \lambda / \sigma\right)}{1-G\left(\varepsilon_{i} \lambda / \sigma\right)}-\frac{\varepsilon_{i} \lambda}{\sigma}\right]
$$

Donde: $\sigma^{2}=\sigma_{u}^{2}+\sigma_{v}^{2}, \lambda=\sigma_{u} / \sigma_{v}, g(\cdot)$ representa la función de densidad de una distribución normal estándar y $\mathrm{G}(\cdot)$ es la función de distribución de una normal estándar. La estimación por máxima verosimilitud de la ecuación 1 proporciona estimadores de los parámetros de la varianza $\sigma_{u}^{2}$ y $\sigma_{v}^{2}$. Así, el índice de ET se calcula como:

$$
\mathrm{TE}_{i}=\exp \left(-\mathrm{E}\left[u_{i} \mid \varepsilon_{i}\right]\right)
$$

La Figura 1 muestra como se calcula la eficiencia técnica en una función de producción. Supongamos que un entrenador $A$ y otro $B$ entrenan al mismo equipo en la misma temporada de esta forma tienen la misma cantidad de input disponible (calidad de plantilla). Para poder comparar el output de los entrenadores es necesario ajustar los puntos conseguidos por los partidos en los que han ejercido de entrenadores. De esta forma en el eje de ordenadas se representan

\footnotetext{
${ }^{3}$ Otras distribuciones utilizadas en la literatura son la exponencial, la normal truncada y la gamma.
} 
los puntos divididos por el máximo número de puntos posibles. A ese equipo le comenzó entrenando el entrenador $A$, teniendo una actuación inferior a lo que esperaban los dirigentes de ese club. La eficiencia del entrenador $A$ se calcula como $y_{A} / y_{B}$ que está entre 0 y 1 . Con el cambio de entrenador se obtiene el efecto deseado y el entrenador $B$ obtiene el número de puntos predichos por la función de producción y por tanto su índice de eficiencia técnica es de $1\left(y_{B} / y_{B}\right)$.

Figura 1

Cálculo de eficiencia técnica en una función de producción

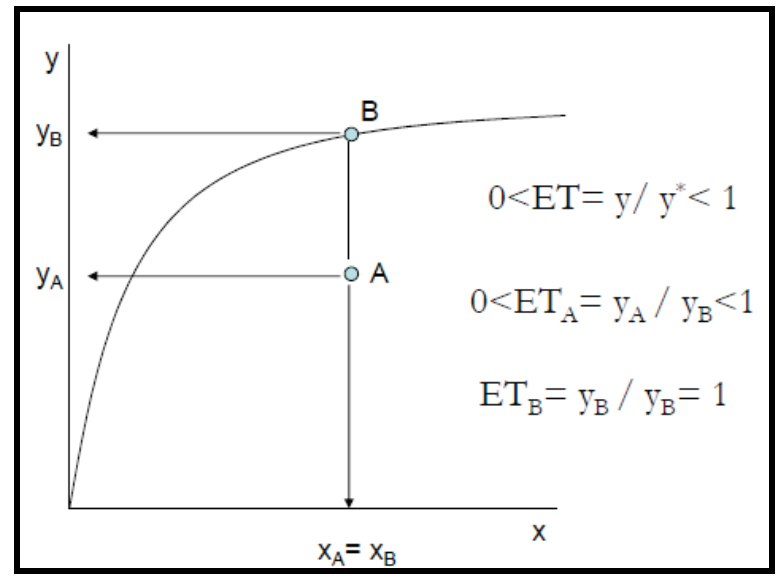

Fuente: Elaboración propia.

Caudill et al. (1995) extendió este modelo para analizar como influyen algunas variables al término de ineficiencia $u_{i}$. De forma específica, estos autores desarrollaron un modelo en el que los determinantes de la ineficiencia son evaluados usando un modelo heterocedástico multiplicativo. Es decir,

$$
\sigma_{u i}=\sigma_{u} \exp \left(Z_{m i} ; \alpha\right)
$$

Donde $Z_{m i}$ es un vector de variables que intentan explicar la ineficiencia y $\alpha$ es un vector de parámetros a estimar. Dado que se asume que la ineficiencia sigue una distribución semi-normal, un incremento de la varianza conlleva incrementos en el nivel de ineficiencia. Por tanto un signo positivo en uno de los parámetros de $\alpha$ significa que esa variable está relacionada de forma negativa con el nivel de eficiencia.

\section{DATOS Y MODELO EMPÍRICO}

Los datos para realizar el estudio provienen de distintas fuentes. En primer lugar como el objetivo del trabajo es estudiar la eficiencia de los entrenadores es necesario conocer quién era el entrenador de los distintos equipos en los parti- 
dos de liga. Para ello se consultó, si era necesario, en Wikipedia las fechas en la que hubo cambios de entrenador. Los resultados de los 760 partidos fueron obtenidos de la web http://www.football-data.co.uk/. Con los resultados de los partidos y quién era el entrenador de cada equipo es relativamente fácil calcular los puntos conseguidos por cada uno de los entrenadores y equipos en las dos temporadas en estudio (2009-2010 y 2010-2011). Lee (2006) argumenta que para evitar problemas de endogeneidad en la estimación de la función de producción de los entrenadores es necesario usar como input una medida de la calidad de la plantilla ex ante.

En este trabajo se propone utilizar el precio de los jugadores en una liga fantástica como es el Comunio. El Comunio es un juego online www.comunio.es en el cual los jugadores (miembros) forman su propia comunidad con el objetivo de ser el que obtenga la máxima puntuación dentro de la comunidad. Cada miembro recibe desde el principio un equipo con 15 jugadores de la Liga BBVA y un presupuesto valorado en 20 millones de EUR, o empieza sin jugadores y recibe un presupuesto valorado en 40 millones de EUR. En cada jornada los jugadores tienen que decidir los 11 jugadores que van a formar parte de su alineación en esa jornada. Después de cada jornada, serán distribuidos puntos a cada jugador de los equipos. La puntuación es calculada por el ordenador según una serie de criterios, incluyendo goles, tarjetas amarillas y rojas y el análisis de un columnista deportivo. En cualquier momento, cada miembro intenta mejorar su presupuesto y su equipo comprando y vendiendo jugadores. De esta forma se establece un mercado de futbolistas en el que Comunio establece un precio inicial y luego es el mercado el que determina el precio de los jugadores. Así, si los miembros de Comunio creen que un jugador está sobrevalorado no serán capaces de venderlo al precio de mercado actual y su precio tenderá a la baja. Lo contrario sucede si los miembros de Comunio creen que un jugador está infravalorado, en este caso el precio del jugador tenderá al alza.

En fútbol, juegan 11 jugadores. De ellos uno es el portero y 10 jugadores "de campo". Los jugadores de campo se clasifican en función de su rol en el campo en defensas, centrocampistas y delanteros. Este orden se corresponde con la cercanía que suelen ocupar en el campo respecto a su propia portería. Un aspecto importante para medir de una forma correcta la calidad de la plantilla, que en la literatura no se ha considerado, es desagregar la calidad de la plantilla por posiciones en el campo. ${ }^{4}$ Los equipos tienen un máximo de 25 jugadores en la plantilla. En general la mayoría de los equipos cubren las 25 licencias disponibles o como máximo dejan sin cubrir 2 ó 3 . Sin embargo, el número de minutos

\footnotetext{
${ }^{4}$ Una excepción es Lee y Berri (2008) donde para analizar la eficiencia de los entrenadores de la NBA incorporan en la función de producción por separado la calidad de los bases, aleros y pivots.
} 
jugados entre los jugadores es muy diferente entre ellos. Hay algunos jugadores que juegan casi todos los partidos completos mientras que hay jugadores que apenas cuentan para sus entrenadores. En la temporada 2010-2011 alrededor del $90 \%$ de los minutos de la liga de cada equipo fueron jugados por sólo 16 jugadores. Por este motivo, para obtener la calidad de la plantilla en cada una de las posiciones se ha calculado el promedio del precio de 1 portero, 6 defensas, 6 centrocampistas y 3 delanteros. Así mismo los precios de intercambio en el Comunio de la temporada 2010-2011 se han transformado para que la media en cada puesto sea la misma en las dos temporadas. De este modo se evita un posible problema de cambio en la valoración entre temporadas.

En las Figuras 2 y 3 puede verse la relación que hay entre la calidad de la plantilla medida como la suma del portero mejor valorado, de 6 defensas, 6 centrocampistas y 3 delanteros y los puntos conseguidos por cada uno de los equipos en ambas temporadas. Puede verse como según este método la plantilla major valorada en las dos temporadas es la del F.C. Barcelona seguido por la del Real Madrid. Los dos siguientes equipos son el Atlético de Madrid y Valencia C.F. en la temporada 2009/2010 la distancia es importante entre las dos primeras plantillas y sus inmediatos perseguidores, pero esta distancia se hace aún mayor en la temporada 2010-2011. Esto se debe en parte a que el Valencia vendió a una de sus estrellas (Villa) al Barcelona.

Figura 2

Relación entre la calidad de la plantilla y puntos en la temporada 2009-2010

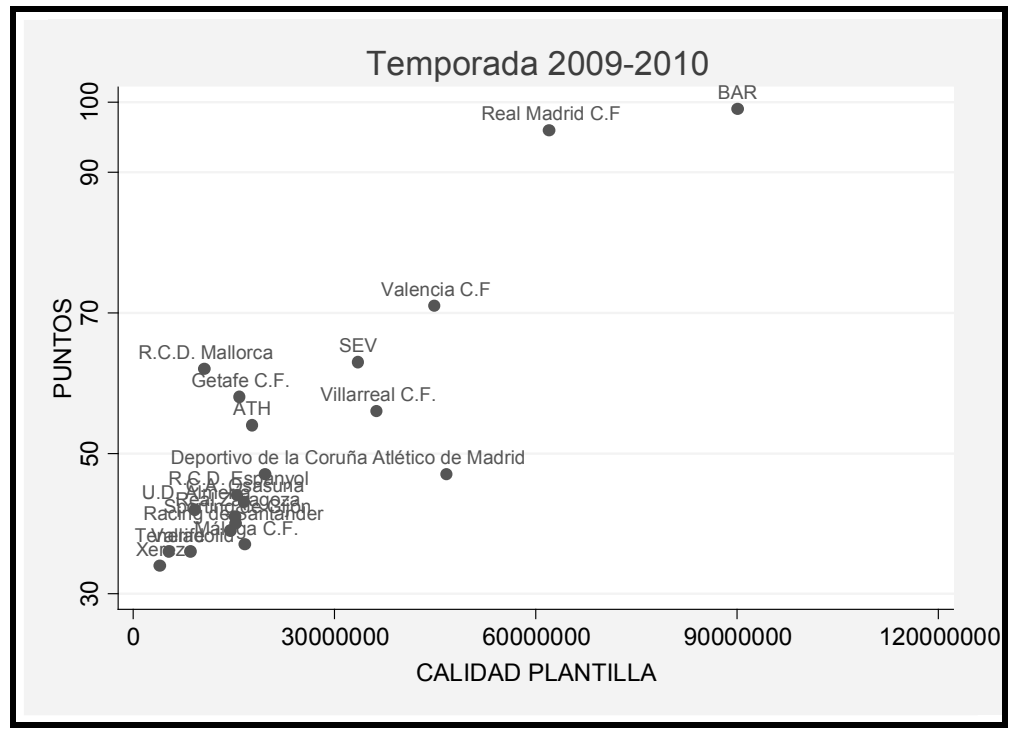

Fuente: Elaboración propia. 
Figura 3

Relación entre la calidad de la plantilla y puntos en la temporada 2010-2011

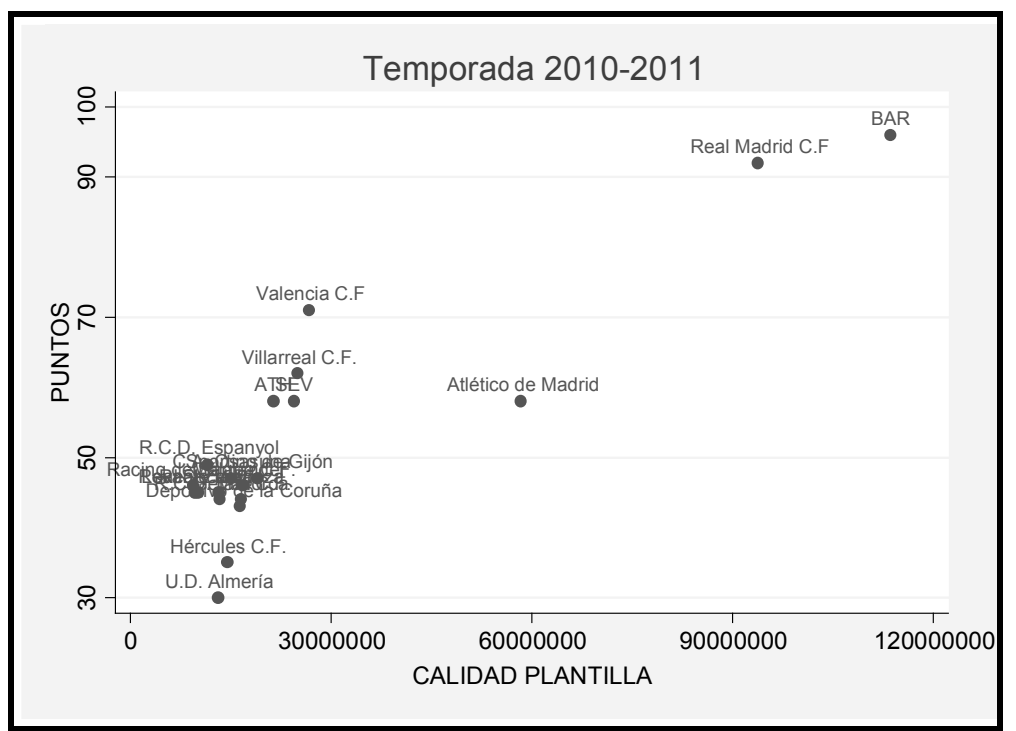

Fuente: Elaboración propia.

El nivel de observación es el entrenador en cada una de las temporadas. De esta forma hay 57 observaciones. No todos los entrenadores en una temporada participan en el mismo número de jornadas dado que si los dirigentes de un equipo perciben que la actuación puede mejorar cambiando al entrenador lo harán. Por tanto para poder comparar a los distintos entrenadores entre sí hay que utilizar un output que no dependa del número de jornadas. Este es el motivo por el cual el output es la ratio entre los puntos conseguidos por un entrenador y el número máximo de puntos que hubiese podido conseguir $\left(3{ }^{*} n^{\circ}\right.$ de jornadas).

La forma funcional de la función de producción es translog. Se ha escogido una forma funcional flexible para no imponer restricciones en la tecnología a estimar. La variable dependiente es la ratio entre puntos conseguidos por un entrenador en una temporada y el número de puntos que hubiese conseguido de haber ganado todos los encuentros. Los inputs utilizados son 4: portero, defensas, centrocampistas y delanteros. Mientras que como variables explicativas de la ineficiencia se utilizan dos variables dummy. La primera de ellas toma el valor 1 si el entrenador fue jugador profesional de fútbol y 0 en caso contrario, mientras que la segunda es una variable dummy que toma valor 1 si el equipo tiene un filial jugando esa temporada en al menos la tercera división del fútbol español ( $\left.2^{\mathrm{a} B}\right)$. Así el modelo a estimar es: 


$$
\begin{aligned}
& \ln y_{i t}=\beta_{0}+\sum_{j=1}^{4} \beta_{j} \ln x_{j i t}+\frac{1}{2} \sum_{j=1}^{4} \sum_{k=1}^{4} \beta_{j k} \ln x_{j i t} \ln x_{k i t}+v_{i t}-u_{i t} ; \\
& u_{i t}=\sigma_{u} \cdot\left(\alpha_{1}+\sum_{z=1}^{2} \alpha_{z} z_{i t}\right)
\end{aligned}
$$

donde $i$ indica entrenador, $t$ denota temporada, $j$ y $k$ son los subíndices de inputs. Se asume que $v$ sigue una distribución, mientras que $u$ sigue una distribución semi-normal. Dado el número tan pequeño de temporadas se ha preferido utilizar un modelo pooled en el que no se tiene en cuenta la existencia de datos de panel.

\section{RESULTADOS}

Como es habitual en la estimación de una forma funcional translog las variables independientes han sido divididas por su media geométrica (e.g., Barros, del Corral y García-del-Barrio, 2008) por lo que los coeficientes de primer orden pueden interpretarse como elasticidades, i.e., el cambio porcentual en el output cuando un input se incrementa en un $1 \% .^{5}$

Las elasticidades output de los input calidad de los defensas $(0,085)$, calidad de los centrocampistas $(0,215)$ son positivas y significativas mientras que la calidad de los delanteros $(0.044)$ es positiva pero no resultó significativa cumpliéndose en estos tres input la propiedad de la tecnología de monotonía. Sin embargo, la elasticidad output del portero es negativa, reflejando posiblemente un problema de multicolinealidad en los datos. La elasticidad de escala (i.e., la suma de todas las elasticidades output) es 0.276 , por tanto existen rendimientos decrecientes a escala. Este hecho indica que si se incrementasen todos los inputs en una determinada proporción el output aumentaría en una proporción menor de lo que han hecho los inputs. Este resultado está en línea con la percepción de que a medida que se aumenta la calidad de la plantilla resulta más complicado aumentar el porcentaje de puntos conseguidos. Este hecho se ve claramente en las Figuras 2 y 3 , donde si uno trazase la función de producción de los equipos en cada una de las temporadas trazaría una función de producción cóncava.

El signo de la variable explicativa de la ineficiencia ${ }^{6}$ "Entrenador Jugador" es positivo y significativo indicando que los entrenadores que fueron futbolistas profesionales fueron menos eficientes. Por último el signo de la variable

\footnotetext{
${ }^{5}$ Se ha realizado el contraste de la forma funcional translog versus la forma funcional Cobb-Douglas rechazando la hipótesis nula de que todos los coeficientes cuadrados y cruzados son iguales a cero, por tanto la forma funcional translog se prefiere a la Cobb-Douglas.

${ }^{6}$ Se ha realizado un test de la ratio de verosimilitudes para contrastar que todos los efectos explicativos de la ineficiencia son cero. El resultado del test sugiere rechazar esta hipótesis.
} 
"Equipo inferior" es negativo pero no significativo indicando que no existe relación entre disponer de buen equipo filial y la eficiencia de los entrenadores.

Tabla 2

Estimación de la función de producción

\begin{tabular}{|c|c|c|}
\hline Variable & Coeficiente & SD \\
\hline Constante & $-0,542^{* * *}$ & $(0,011)$ \\
\hline Portero & $-0,068^{\star * *}$ & $(0,016)$ \\
\hline Defensas & $0,085^{\star * *}$ & $(0,009)$ \\
\hline Centrocampistas & $0,215^{* * *}$ & $(0,064)$ \\
\hline Delanteros & 0,044 & $(0,046)$ \\
\hline Portero×Portero & $-0,160^{\star * *}$ & $(0,017)$ \\
\hline Defensas×Defensas & $-0,411^{* *}$ & $(0,193)$ \\
\hline Centrocampista×Centrocampista & $-0,141$ & $(0,306)$ \\
\hline Delantero×Delantero & $-0,382^{* * *}$ & $(0,127)$ \\
\hline Portero×Defensas & $0,252^{\star * *}$ & $(0,076)$ \\
\hline Portero×Centrocampistas & $-0,056$ & $(0,041)$ \\
\hline Portero×Delanteros & $-0,018$ & $(0,045)$ \\
\hline Defensas $\times$ Centrocampistas & $-0,245$ & $(0,218)$ \\
\hline Defensas $\times$ Delanteros & 0,076 & $(0,057)$ \\
\hline Centrocampistas $\times$ Delanteros & $0,525^{\star * *}$ & $(0,081)$ \\
\hline \multicolumn{3}{|l|}{ Modelo de ineficiencia } \\
\hline Constante & $-2,492^{* * *}$ & $(0,424)$ \\
\hline Entrenador jugador & $1.204^{* * *}$ & $(0,427)$ \\
\hline Equipo inferior & -0.086 & $(0,387)$ \\
\hline$\sigma_{u}$ & 0,461 & \\
\hline$\sigma_{v}$ & 0,000 & \\
\hline Log-likelihood & 5,778 & \\
\hline
\end{tabular}

Fuente: Elaboración propia.

Una vez calculada la función de producción de los entrenadores es posible calcular la eficiencia técnica de los entrenadores. La eficiencia técnica de los entrenadores en cada una de las dos temporadas se muestra en las Tabla 3 (2009-2010) y Tabla 4 (2010-2011). La suma del número de partidos dirigidos por los entrenadores es de 760 para la temporada $2009-2010$ y de 759 para la temporada 2010-2011. Esto se debe a que en la temporada 2010-2011 el Málaga utilizó a un entrenador sólo una jornada, entrenador que no ha sido incluido en las estimaciones. Estudios previos han encontrado una clara correlación positiva entre la eficiencia de los entrenadores y permanencia en el puesto (Scully, 1994; 
De Dios Tena y Forrest, 2007; Fort, Lee y Berri, 2008; Volz, 2009). Este mismo resultado se encuentra aquí donde los entrenadores más ineficientes en ambas temporadas fueron despedidos.

Tabla 3

ET de los entrenadores en la temporada 2009-2010

\begin{tabular}{|c|c|c|c|c|c|}
\hline Entrenador & Equipo & partidos & puntos & $\%$ puntos & ET \\
\hline Camacho & C.A. Osasuna & 38 & 43 & $38 \%$ & 1.00 \\
\hline Clemente & Real Valladolid C.F. & 8 & 12 & $50 \%$ & 1.00 \\
\hline Garrido & Villarreal C.F. & 18 & 30 & $56 \%$ & 1.00 \\
\hline Guardiola & F.C. Barcelona & 38 & 99 & $87 \%$ & 1.00 \\
\hline Lillo & U.D. Almería & 20 & 27 & $45 \%$ & 1.00 \\
\hline Lotina & Deportivo de la Coruña & 38 & 47 & $41 \%$ & 1.00 \\
\hline Manzano & R.C.D. Mallorca & 38 & 62 & $54 \%$ & 1.00 \\
\hline Michel & Getafe C.F. & 38 & 58 & $51 \%$ & 1.00 \\
\hline Pellegrini & Real Madrid C.F & 38 & 96 & $84 \%$ & 0.99 \\
\hline Antonio Álvarez & Sevilla F.C. & 10 & 18 & $60 \%$ & 0.89 \\
\hline Gorosito & Xerez C.D. & 21 & 27 & $43 \%$ & 0.86 \\
\hline Quique & Atlético de Madrid & 31 & 41 & $44 \%$ & 0.86 \\
\hline Juan Ramón López & Málaga C.F. & 38 & 37 & $32 \%$ & 0.82 \\
\hline Caparrós & Athletic de Bilbao & 38 & 54 & $47 \%$ & 0.82 \\
\hline Jiménez & Sevilla F.C. & 28 & 45 & $54 \%$ & 0.79 \\
\hline Valverde & Villarreal C.F. & 20 & 26 & $43 \%$ & 0.78 \\
\hline Emery & Valencia C.F & 38 & 71 & $62 \%$ & 0.77 \\
\hline Portugal & Racing de Santander & 28 & 32 & $38 \%$ & 0.75 \\
\hline Pochettino & R.C.D. Espanyol & 38 & 44 & $39 \%$ & 0.74 \\
\hline Oltra & C.D. Tenerife & 38 & 36 & $32 \%$ & 0.72 \\
\hline Preciado & Sporting de Gijón & 38 & 40 & $35 \%$ & 0.64 \\
\hline Hugo Sánchez & U.D. Almería & 18 & 15 & $28 \%$ & 0.62 \\
\hline Gay & Real Zaragoza & 24 & 29 & $40 \%$ & 0.60 \\
\hline Mendilibar & Real Valladolid C.F. & 19 & 17 & $30 \%$ & 0.60 \\
\hline Abel & Atlético de Madrid & 7 & 6 & $29 \%$ & 0.56 \\
\hline Mandiá & Racing de Santander & 10 & 7 & $23 \%$ & 0.46 \\
\hline Marcelino & Real Zaragoza & 14 & 12 & $29 \%$ & 0.43 \\
\hline Onésimo & Real Valladolid C.F. & 11 & 7 & $21 \%$ & 0.42 \\
\hline Ziganda & Xerez C.D. & 17 & 7 & $14 \%$ & 0.28 \\
\hline
\end{tabular}

Nota: Los entrenadores en cursiva fueron despedidos.

Fuente: Elaboración propia. 
Tabla 4

ET de los entrenadores en la temporada 2010-2011

\begin{tabular}{|c|c|c|c|c|c|}
\hline Entrenador & Equipo & Partidos & Puntos & $\%$ puntos & ET \\
\hline Aguirre & Real Zaragoza & 28 & 38 & $45 \%$ & 1.00 \\
\hline Emery & Valencia C.F & 38 & 71 & $62 \%$ & 1.00 \\
\hline Marcelino & Racing de Santander & 16 & 24 & $50 \%$ & 1.00 \\
\hline Mendilibar & C.A. Osasuna & 15 & 25 & $56 \%$ & 1.00 \\
\hline Pellegrini & Málaga C.F. & 28 & 39 & $46 \%$ & 1.00 \\
\hline Quique & Atlético de Madrid & 38 & 58 & $51 \%$ & 0.95 \\
\hline Djukic & Hércules C.F. & 9 & 9 & $33 \%$ & 0.95 \\
\hline Caparrós & Athletic de Bilbao & 38 & 58 & $51 \%$ & 0.94 \\
\hline Pochettino & R.C.D. Espanyol & 38 & 49 & $43 \%$ & 0.91 \\
\hline Mourinho & Real Madrid C.F & 38 & 92 & $81 \%$ & 0.91 \\
\hline Luis García & Levante U.D. & 38 & 45 & $39 \%$ & 0.88 \\
\hline Esteban Vigo & Hércules C.F. & 29 & 26 & $30 \%$ & 0.85 \\
\hline Antonio Álvarez & Sevilla F.C. & 5 & 8 & $53 \%$ & 0.85 \\
\hline Guardiola & F.C. Barcelona & 38 & 96 & $84 \%$ & 0.83 \\
\hline Garrido & Villarreal C.F. & 38 & 62 & $54 \%$ & 0.82 \\
\hline Lasarte & Real Sociedad & 38 & 45 & $39 \%$ & 0.81 \\
\hline Manzano & Sevilla F.C. & 33 & 50 & $51 \%$ & 0.80 \\
\hline Laudrup & R.C.D. Mallorca & 38 & 44 & $39 \%$ & 0.80 \\
\hline Oltra & U.D. Almería & 20 & 20 & $33 \%$ & 0.79 \\
\hline Preciado & Sporting de Gijón & 38 & 47 & $41 \%$ & 0.76 \\
\hline Lotina & Deportivo de la Coruña & 38 & 43 & $38 \%$ & 0.69 \\
\hline Michel & Getafe C.F. & 38 & 44 & $39 \%$ & 0.68 \\
\hline Portugal & Racing de Santander & 22 & 22 & $33 \%$ & 0.67 \\
\hline Lillo & U.D. Almería & 12 & 9 & $25 \%$ & 0.59 \\
\hline Camacho & C.A. Osasuna & 23 & 22 & $32 \%$ & 0.57 \\
\hline Gay & Real Zaragoza & 10 & 7 & $23 \%$ & 0.52 \\
\hline Jesualdo Ferreira & Málaga C.F. & 9 & 7 & $26 \%$ & 0.48 \\
\hline
\end{tabular}

Nota: Los entrenadores en cursiva fueron despedidos.

Fuente: Elaboración propia.

Hay que matizar que este índice solo tiene en cuenta la actuación en la liga española. Sin embargo los clubes no sólo compiten en la liga sino que también compiten en competiciones europeas y la Copa del Rey. Este hecho resulta de especial importancia por ejemplo para el entrenador del Villarreal en la temporada 2010-2011, equipo que una vez garantizada su plaza de Champions League dejó la liga un poco de lado y se centro en la Europa League. Por lo tanto, todo parece indicar que el nivel de eficiencia de Garrido está sesgado a la baja. 
Una pregunta interesante es si el cambio de entrenador resulta positivo en el caso de que el equipo lleve una mala trayectoria. A esta pregunta la responde de forma afirmativa Gónzalez-Gómez, Picazo-Tadeo y García-Rubio (2011). En este trabajo estiman un modelo DEA para la Liga de Primera división del Fútbol español entre las temporadas 2001/2002 y 2008/2009 para luego comparar la eficiencia de tres grupos distintos (los entrenadores que están toda la temporada, los entrenadores que son despedidos y los entrenadores que son contratados a mitad de temporada) usando test de diferencia de medias. La Tabla 5 muestra la eficiencia técnica de los entrenadores utilizando la misma clasificación que Gónzalez-Gómez, Picazo-Tadeo y García-Rubio (2011) en las dos temporadas en estudio.

\section{Tabla 5}

ET media de los entrenadores en función de la temporada y permanencia

\begin{tabular}{lccc}
\hline & $\mathbf{2 0 0 9 / 2 0 1 0}$ & $\mathbf{2 0 1 0 / 2 0 1 1}$ & Total \\
\hline Entrenadores cesados & 0.55 & 0.63 & 0.59 \\
Entrenadores con 38 partidos & 0.87 & 0.86 & 0.86 \\
Entrenadores contratados en mitad de temporada & 0.91 & 0.92 & 0.92 \\
\hline
\end{tabular}

Fuente: Elaboración propia.

Se puede ver como el grupo de entrenadores más eficiente en las dos temporadas es el grupo de entrenadores contratados en mitad de temporada (y no despedidos) con una eficiente técnica media de 0,92 , seguido de los entrenadores que estuvieron las 38 jornadas del campeonato liguero al frente de sus equipos con una eficiencia técnica media de 0,86 . El grupo más ineficiente con diferencia es el de entrenadores despedidos con un índice de eficiencia técnica media de 0,59. Para ver si estas diferencias son estadísticamente significativas se ha realizado un t-test de diferencia entre los entrenadores despedidos y los entrenadores contratados. Los resultados sugieren que las diferencias entre estos dos grupos han resultado significativas en ambas temporadas dado que el $p$-value es menor que 0,01 . De este modo se obtienen resultados similares a los obtenidos en el trabajo de Gónzalez-Gómez, Picazo-Tadeo y García-Rubio (2011) para las dos temporadas siguientes.

Por último en la Tabla 6 se analiza la media de la eficiencia técnica de los entrenadores en función de si tienen pasado como futbolistas profesionales o no. Los resultados muestran que los entrenadores que no han sido futbolistas profesionales son más eficientes. Una posible explicación es que los entrenadores que no han sido futbolistas profesionales y llegan a entrenar en la Primera División es que han demostrado su valía previamente en otras categorías mientras que muchos de los entrenadores que han sido futbolistas profesionales no cumplen 
este requisito y haber sido buen jugador no es garantía de ser buen entrenador, desdiciendo el popular refrán español "para ser un buen fraile hay que ser antes cocinero". En cualquier caso estos resultados deben tomarse con cierta cautela dado que el número de temporadas usado es bajo, y por tanto una posible ampliación del trabajo consistiría en una ampliación de la base de datos para contrastar esta hipótesis con una mayor robustez.

Tabla 6

ET media de los entrenadores en función de la temporada y pasado como futbolista profesional

\begin{tabular}{lccc}
\hline & $\mathbf{2 0 0 9 / 2 0 1 0}$ & $\mathbf{2 0 1 0 / 2 0 1 1}$ & Total \\
\hline No jugador profesional & 0.91 & 0.81 & 0.85 \\
Jugador profesional & 0.73 & 0.79 & 0.76 \\
\hline
\end{tabular}

Fuente: Elaboración propia.

\section{CONCLUSIONES}

Existen numerosos trabajos que han analizado la eficiencia de los entrenadores en la literatura. Aunque está comúnmente aceptado por todos los investigadores que debe utilizarse una medida de la calidad ex ante de la plantilla no existe un consenso sobre como hacerlo. En este trabajo se presenta una alternativa a las medidas utilizadas hasta la fecha. En concreto se utilizan los precios de los jugadores en una Liga Fantástica como es el Comunio para analizar la eficiencia de los entrenadores del fútbol español durante las temporadas 2009/2010 y 2010/2011.

Para ello se utiliza un modelo de frontera estocástica utilizando como inputs la calidad de los porteros, defensas, centrocampistas y delanteros por separado. La función de producción muestra que, cuanto mejores sean los jugadores mayor será el output. Así mismo la función de producción se caracteriza por rendimientos decrecientes a escala, lo cual es esperado dado que existe un límite en la puntuación. Los niveles de eficiencia obtenidos muestran que los entrenadores con un índice muy bajo fueron despedidos y más importante que los entrenadores que remplazaron a los entrenadores despedidos tuvieron un rendimiento muy alto. Hecho corroborado con un test de diferencia de medias. Por tanto, el cambio de entrenador resultó positivo en media en estas dos temporadas. 


\section{REFERENCIAS BIBLIOGRÁFICAS}

AIGNER, D., LOVELL, C.A.K. y SCHMIDT, P. (1977). "Formulation and Estimation of Stochastic Frontier Production Function Models", en Journal of Econometrics, 6, pp. 21-37.

ÁlVAREZ, A. (2001). La medición de la eficiencia y la productividad, Pirámide, Madrid.

BARROS, C.P., DEL CORRAL, J. y GARCIA-DEL-BARRIO, P. (2008). "Identification of segments of soccer clubs in the Spanish League First Division with a latent class model" en Journal of Sports Economics, 9(5), pp. 451-469.

BARROS, C.P. y GARCIA-DEL-BARRIO, P. (2011). "Productivity drivers and market dynamics in the Spanish First Division football league" en Journal of Sports Economics, 35, pp. 5-13.

BARROS, C.P. y LEACH, S. (2007). "Technical efficiency in the English Football Association Premier League with a stochastic cost frontier" en Applied Economics Letters, 14, pp. 731-741.

BOSCÁ, J., LIERN, V., MARTÍNEZ, A. y SALA, R. (2009). "Increasing offensive or defensive efficiency? An analysis of Italian and Spanish football" en Omega, 37, pp.63-78.

CARMICHAEL, F. y THOMAS, D. (1995). "Production and efficiency in sports: an investigation of rugby league football" en Applied Economics, 27, pp. 859-869.

CARMICHAEL, F., THOMAS, D. y WARD, R. (2001). "Production and efficiency in Association Football" en Journal of Sports Economics, 2(3), pp. 228-243.

CAUDILL, S. B., FORD, J. M. y GROPPER, D. M. (1995). "Frontier estimation and firm-specific inefficiency measures in the presence of heteroscedasticity" en Journal of Business and Economic Statistics, 13, pp. 105-111.

COLLIER, T., JOHNSON, A.L. y RUGGIERO, J. (2011). "Measuring technical efficiency in sports" en Journal of Sports Economics, 12, pp. 579-598.

DAWSON, P., DOBSON, S. y GERRARD, B. (2000a). "Estimating coaching efficiency in professional team sports: evidence from English Association Football” en Scottish Journal of Political Economy, 47(4), pp. 399-421.

DAWSON, P., DOBSON, S. y GERRARD, B. (2000b). "Stochastic frontiers and the temporal structure of managerial efficiency in English soccer" en Journal of Sports Economics, 1, 341-362.

DIOS PALOMARES, R., MARTÍNEZ PAZ, J.M. y VICARIO MODROÑO, V. (2003). "Eficiencia versus innovación en explotaciones agrarias" en Estudios de Economía Aplicada, 21(3), pp. 485-501.

DE DIOS TENA, J. y FORREST, D. (2007). "Within-season dismissal of football coaches: Statistical analysis of causes and consequences" en European Journal of Operations Research, 181, pp. 362-373. 
EINOLF, K.W. (2004). "Is winning everything? A Data Envelopment Analysis of Major League Baseball and National Football League" en Journal of Sports Economics, 5(2), pp. 127-151.

ESPITIA-ESCUER, M. y GARCÍA-CEBRIÁN, L.I. (2004). "Measuring the efficiency of Spanish First-Division Soccer Teams" en Journal of Sports Economics, 5, pp. 329-346.

FIZEL, J.L. y D'ITRI, M.P. (1997). "Managerial efficiency, managerial succession and organizational performance" en Managerial and Decision Economics, 18, pp. 295-308.

FORT, R., LEE, Y.H. y BERRI, D. (2008). "Race, technical efficiency, and retention: The case of NBA coaches" en International Journal of Sport Finance, 3, pp. 84-97.

FRICK, B. y SIMMONS, R. (2008). "The impact of managerial quality on organizational performance: Evidence from German soccer" en Managerial and Decision Economics, 29, pp. 593-600.

GARCÍA-SÁNCHEZ, I.M. (2007). "Efficiency and effectiveness of Spanish football teams: a three-stage-DEA approach" en Central European Journal of Operations Research, 15, pp. 21-45.

GERRARD, B. (2001). "A new approach to measuring player and team quality in professional team sports" en European Sport Management Quarterly, 1, pp. 219-233.

GINER, C. y MUÑOZ, A. (2008). “¿Son los clubes de fútbol eficientes? Aplicación del análisis DEA a los equipos de la liga profesional de fútbol de España", en Universia Business Review, 17, pp. 12-25.

GONZÁLEZ-GÓMEZ, F. y PICAZO-TADEO, A.J. (2010). "Can we satisfied with our football team" en Journal of Sports Economics, 11, pp. 418-442.

GONZÁLEZ GÓMEZ, F., PICAZO TADEO, A.J. y GARCÍA-RUBIO, M.A. (2011). "Impact of a mid-season change of manager on sporting performance" en Sport, Business and Management, 1, pp. 28-42.

HADLEY, L., POITRAS, M., RUGGIERO, J. y KNOWLES, S. (2000). "Performance evaluation of National Football League clubs" en Managerial and Decision Economics, 21, pp. 63-70.

HOFLER, R.A. y PAYNE, J.E. (1997). "Measuring efficiency in the National Basketball Association" en Economics Letters, 55, pp. 293-299.

JONDROW, J., LOVELL, C.A.K., MATEROV, I.S. y SCHMIDT, P. (1982). "On the estimation of technical inefficiency in the stochastic frontier production model" en Journal of Econometrics, 19, pp. 233-238.

KAHANE, L.H. (2005). "Production efficiency and discriminatory hiring practices in the National Hockey League: a stochastic frontier approach" en Review of Industrial Organization, 27(1), pp. 47-71.

KERN, M. Y SÜSSMUTH, B. (2008). "Managerial efficiency in German top League Soccer: An econometric analysis of club performances on and off the pitch" en German Economic Review, 6, pp. 485-506. 
KUMBHAKAR, S. y LOVELL, K. (2000). Stochastic Frontier Analysis. Cambridge Univ. Press, Cambridge, UK.

LEE, Y.H. (2006). Team sports efficiency estimation and stochastic frontier models." In John Fizel (ed.) The Handbook of Sports Economics Research. Armonk, NY: M.E. Sharpe, Inc.).

LEE, Y.H. y BERRI, D. (2008). "A re-examination of production functions and efficiency estimates for the National Basketball Association" en Scottish Journal of Political Economy, 55, pp. 51-66.

MEEUSEN, W. y VAN DEN BROECK, J. (1977). "Efficiency Estimation from Cobb-Douglas production functions with composed error" en International Economic Review, 18, pp. 435-444.

PICAZO-TADEO, A.J. y GONZÁLEZ-GÓMEZ, F. (2010). "Does playing several competitions influence a team's league performance? Evidence from Spanish professional football" en Central European Journal of Operations Research, 18, pp. 413-432.

PORTER, P.K. y SCULLY, G.W. (1982). "Measuring managerial efficiency: the case of baseball" en Southern Economic Journal, 48, pp. 642-650.

SALA-GARRIDO, R., LIERN, V., MARTíNEZ, A. y BOSCÁ, J. (2009). "Analysis and evolution of efficiency in the Spanish Soccer League (2000/01-2007/08)" en Journal of Quantitative Analysis in Sports, 5.

SCULLY, G.W. (1994). "Managerial effiiciency and survivability in profesional team sports" en Managerial and Decision Economics, 15, pp. 403-411.

VOLZ, B. (2009). "Minority status and managerial survival in major league baseball" en Journal of Sports Economics, 10, pp. 522-542.

ZAK, T.A., HUANG, C.J. y SIEGFRIED, J.J. (1979). "Production efficiency: the case of professional basketball" en Journal of Business, 52, pp. 379-392. 
